\title{
Elaboración de informativos de televisión como método docente
}

\author{
Sanjuán Pérez, Antonio ${ }^{1}$; Videla Rodríguez, \\ José Juan²; Martínez Costa, Sandra \\ Universidade da Coruña, Facultade de Ciencias da Comunicación, \\ Códigos ORCID: ${ }^{10000-0001-7612-2838, ~}{ }^{20000-0001-8656-9297 ~}$
}

\section{RESUMEN}

Durante el curso 2017-2018, en la materia Xornalismo Audiovisual se planteó una nueva metodología en la que cada grupo de prácticas elaboraba un informativo realizado en directo y subido a un canal de Youtube (xornalismoudc). La dinámica de trabajo era idéntica a la utilizada en una redacción profesional: se repartía la agenda informativa, los medios técnicos y en la clase siguiente se comenzaba con una reunión de escaleta, minutado y presentación de las piezas y reportajes. La práctica concluía con una mesa de redacción para las previsiones informativas del día siguiente. Las sesiones magistrales se sustituyeron por el visionado en común de los informativos de los respectivos grupos de prácticas. La aplicación de la teoría a la práctica era constante. En las primeras semanas se ensayaron elementos de redacción, locución y elaboración de escaleta sin noticias originales. Quedó así de manifiesto que el proceso de aprendizaje se acelera notablemente cuando el alumno se enfrenta al miedo escénico de la cámara con sensación de realidad y exposición pública, y la consiguiente exigencia de credibilidad y rigor. Igualmente se comprobó que se entienden mejor los procesos haciendo y fallando (learning by doing) que ensayando dentro del marco más conservador del aula.

PALABRAS CLAVE: innovación docente; producción informativa; periodismo audiovisual; aprendizaje experiencial. 


\section{CITA RECOMENDADA:}

Sanjuán Pérez, Antonio; Videla Rodríguez, José Juan; Martínez Costa, Sandra (2020): Elaboración de informativos de televisión como método docente. En De la Torre Fernández, E. (ed.) (2020). Contextos universitarios transformadores: Boas prácticas no marco dos GID. IV Xornadas de Innovación Docente. Cufie. Universidade da Coruña. A Coruña (págs. 239 -250).

DOI capítulo: https://doi.org/10.17979/spudc.9788497497756.239

DOl libro: https://doi.org/10.17979/spudc.9788497497756

\section{ABSTRACT}

During the 2017-2018 academic degree, in the Audiovisual Journalism subject, a new methodology was proposed: each practice group made a live News program and uploaded it to a YouTube channel (xornalismoudc). The work dynamics were identical to those used in professional news department: the informative agenda and technical requirements were distributed, and in the following class it began with a meeting of the running order, timing, and presentation of the pieces and reports. The practice concluded with a drafting table for the informative forecasts of the following day. The master classes were replaced by the public viewing of the news made by the respective practice groups. The application of theory to practice was constant. In the first weeks, also elements of writing, speech, and elaboration of news running order were practiced without real news. It was thus clear that the learning process accelerates markedly when the student faces their stage fright with a sense of reality and public exposure and the consequent demand for credibility and rigor. It was also found that he understands better the processes by doing and failing (learning by doing) than by rehearsing within the most conservative classroom setting.

KEY WORDS: educational innovation; journalism production; audio-visual journalism; project based learning. 


\section{INTRODUCCIÓN}

Las experiencias de innovación en la enseñanza superior suponen la planificación de la docencia utilizando nuevos conocimientos o usos diferentes del conocimiento, que van desde la definición del problema hasta la resolución del mismo. Ello implica, de forma muy habitual, que el docente recurrirá de forma creativa a teorías, concepciones, prácticas o tecnologías adecuadas para alcanzar la resolución del problema de la transmisión y adquisición del conocimiento (Aguiar, B.; Velázquez, R.; Aguiar, J., 2018). La metodología utilizada en esta experiencia de innovación docente, llevada a cabo durante el curso académico 2017-2018, se fundamenta en el aprendizaje experiencial o project based learning (PBL), proceso de enseñanza que consiste en la realización de proyectos y que promueve en el estudiante un aprendizaje activo, crítico y creativo al ser él quien se implica en mayor medida en su propio proceso de formación (Marti, J. A.; Heydrich, M.; Rojas, M.; Hernández, A., 2010). Igualmente, es fundamental en este proceso el llamado learning by doing, metodología docente que se basa en el aprendizaje "haciendo", pero que de forma más específica puede ayudar en la adquisición de conocimientos a través de la corrección de los errores propios y ajenos (GilLacruz, M.; Gracia-Pérez, M.L.; Gil-Lacruz, A.I., 2019).

Este proyecto de innovación docente se centró también en el aprendizaje colaborativo, en el que cada uno de los estudiantes se hace responsable de su propio trabajo y del de los demás (Badía, A.; García, B., 2010). Ello implica que los alumnos trabajen apoyándose unos a otros, aprendiendo a escucharse y a valorar las propuestas de los demás de una manera crítica y constructiva, con el fin de obtener un aprendizaje conjunto más completo y que mejore el trabajo individual. La práctica aquí propuesta favorece también la conexión del estudiante con su entorno social, dado que el producto realizado necesitaba del contacto del alumno con su contexto de actualidad y dado también que este fue mostrado al público en las redes sociales. Esta exposición pública, así como la inmediatez a la que se sometieron los estudiantes con la realización del trabajo es parte fundamental de la formación en los nuevos medios, necesaria para el actual desarrollo de la profesión periodística (Machado, E. \& Teixeira, T., 2016). 
En el contexto de la formación en periodismo, la educación debe adaptarse a los cambios culturales y tecnológicos de las últimas décadas, en especial con la explosión de las redes sociales y del acceso a la información de la ciudadanía. En el marco tecnológico, la formación en nuevos formatos 0 soportes no debe alejar a los estudiantes de la formación sobre las señas de identidad del periodismo (Casals, M.J., 2006); en el campo cultural y social, la formación ha de centrarse en el sentido de la profesión y en su función mediadora y moldeadora de la realidad social. Por tanto, la formación periodística ha de centrarse en la calidad del producto más que en la técnica, en el contraste de fuentes, y en la evitación del espectáculo informativo 0 en la titularización o producción de la noticia en función de sus posibles éxitos de audiencia. Debe formarse al estudiante para la realización de contenido informativo al servicio de su comunidad, aunque, eso sí, sin olvidar los cambios y evoluciones sociales que hacen que, actualmente, se dé "un mapa periodístico complejo, que ha fragmentado las audiencias y ha roto definitivamente el oligopolio en el ámbito de la distribución y la producción por parte de las grandes cabeceras periodísticas" (Manfredi, J. L.; Ufarte, M. J. \& Herranz, J. M., 2019: 1639).

\section{DESCRIPCIÓN DE LA EXPERIENCIA DOCENTE}

\subsection{CONTEXTUALIZACIÓN}

La asignatura de Periodismo Audiovisual se imparte en el primer cuatrimestre del segundo curso de Comunicación Audiovisual de la Universidade da Coruña. Es obligatoria, consta de 6 créditos ECTS, y para conseguir la cualificación de apto, el alumno ha de realizar una serie de trabajos prácticos que indiquen que ha alcanzado las destrezas suficientes para desenvolverse con soltura en un medio informativo. Por ello, deben hacer una serie de ejercicios de redacción y locución, elaborar noticias audiovisuales, reportajes, y editar un programa informativo en directo. La realización de las prácticas permitía al estudiante, en el curso 20172018, conseguir los 10 puntos del total de la materia, pero aquellos que no lo lograsen podrían 
realizar un examen de tipo test con los contenidos teóricos y la actualidad informativa, más un examen práctico final.

La materia se centra en la elaboración de información en el contexto audiovisual y en educar al estudiante en la creación de contenidos de código informativo, y se imparte en el mismo cuatrimestre que otras de formación sobre el código publicitario (Publicidade Audiovisual) y el código de entretenimiento (Ficción Audiovisual). El cuatrimestre se completa con estudios sobre las audiencias (Estudos das Audiencias) y la asignatura de Comunicación Corporativa.

\subsection{DESCRIPCIÓN DE LA PRÁCTICA DE INNOVACIÓN DOCENTE}

El trabajo de innovación docente aquí presentado consistió en la edición y elaboración de los contenidos de un informativo en directo como parte de la práctica de la materia. Para la realización del trabajo el procedimiento fue el siguiente:

1. Los estudiantes se repartían en los grupos medianos asignados por el centro para cada una de las sesiones prácticas de dos horas de duración. Cada grupo tenía una de esas sesiones a la semana.

2. A los estudiantes se les encargaba la realización de una pieza informativa, de entre 60 segundos y un minuto y medio, de contenido de actualidad y relacionado con el entorno más cercano al estudiante: A Coruña y alrededores, y la propia Universidad. En la evaluación de las noticias primaba la pertinencia y la actualidad de las mismas, por lo que lo ideal era que el estudiante los realizase en las horas previas a la edición del informativo. Para fijar los temas del informativo se hacía un reparto previo de contenidos y de necesidades técnicas y materiales la semana anterior.

3. La pieza debía contar con al menos cuatro "totales" (cortes de declaraciones relacionadas con la información).

4. En la sesión práctica semanal uno de los estudiantes se convertía en el editor del informativo y, por lo tanto, en el que decidía el orden de emisión de los contenidos 0 
cuáles de ellos se quedaban fuera en el caso de que hubiese dos noticias semejantes. La preparación del informativo duraba en torno a 20-30 minutos.

5. Cada uno de los estudiantes debía presentar y locutar ante las cámaras su noticia. Además, uno de los docentes de la materia, con años de experiencia como periodista y locutor radiofónico, participaba también como presentador del informativo. En la realización en directo colaboraba otra docente del centro, y alguno de los estudiantes que ayudaban como soporte a la hora de "pinchar" las entradas de vídeo o los micrófonos del directo. Las cortinillas, cabeceras y rótulos fueron previamente diseñados por el profesor responsable de la materia. Para la edición en directo del programa se utilizó un dispositivo portátil Atem Television Studio, que permite la sincronización de 6 fuentes distintas de las cuales dos eran cámaras robotizadas (controladas de forma remota). La visualización del directo se hacía en un ordenador portátil y a su vez se utilizaba también un segundo portátil a modo de prompteri. El dispositivo portátil permitía que la realización del directo se hiciese en cualquier espacio, lo que daba una mayor flexibilidad de la que daría la utilización del plató con el que cuenta la Facultade de Ciencias da Comunicación. Por ello, y dado que el espacio del plató estaba ocupado en las fechas de las prácticas, los informativos se hicieron en una de las salas de la biblioteca, un espacio más abierto, expuesto al público y diáfano que el aula.

La realización del directo se grababa para su posterior subida a las redes sociales, en concreto al canal de Youtube de la materia. Al ser los docentes los encargados de la realización técnica en esta fase del trabajo, el estudiante debía preocuparse únicamente por la elaboración del contenido periodístico, lo que evitaba que se dispersase con los problemas técnicos propios de la realización (a los que se enfrentan en la materia de Realización Audiovisual impartida el cuarto curso). Para la creación de las piezas los estudiantes podían utilizar sus teléfonos móviles tanto a 
modo de cámaras como de micrófonos, lo que a su vez suponía que trabajaban con una tecnología perfectamente conocida por ellos.

6. La edición en directo finalizaba con un informativo de entre 15 y 30 minutos que se subía al canal de Youtube de la asignatura (xornalismoudc). Cada uno de los grupos realizó 6 informativos, lo que hizo un total de 18, y una suma de 261 piezas para el curso académico 2017-18.

7. La evaluación de la intervención de los estudiantes en el programa en directo y de las piezas editadas previamente para el informativo la hacían los profesores responsables de la materia durante la realización del mismo.

8. Tras la elaboración del directo, los estudiantes se reunían para preparar las previsiones informativas de la siguiente clase.

9. Por último, se pedía a cada alumno que autoevaluase sus propias piezas y locuciones en directo. Además de ellos, cada uno de los tres profesores que intervinieron en las prácticas, evaluaba por separado cada pieza y cada locución. La nota final de la materia se completaba con un test sobre los contenidos teóricos y que se habían facilitado a los estudiantes a través de la plataforma Moodle, más un test de contenidos de actualidad informativa. Aquellos estudiantes que no participasen del sistema de evaluación continua tenían la posibilidad de presentarse a un examen final con el test de contenidos docentes y de actualidad, más una práctica de redacción y locución ante cámara.

Del proceso de trabajo aquí definido se deduce que la dinámica de trabajo era, por lo tanto, idéntica a la utilizada en una redacción profesional, ya que había un primer reparto de la agenda informativa, con las previsiones de los temas del noticiario de la siguiente clase y la elaboración de la escaleta, el minutado y la presentación de las piezas y los reportajes el día de la realización. Resumiendo, el proceso empleado seguía por lo tanto las fases más habituales en la producción de noticias, concretadas por Domingo, D., Quandt, T., Heinonen, A., Paulussen, S., Singer, J. B., \& Vujnovic, M., (2008): 
1. Observación del entorno para la detección de la noticia y su relevancia.

2. Selección y filtrado de la noticia, proceso que se llevaba a cabo tanto por el profesor responsable de la materia como por el estudiante "editor" de cada grupo de trabajo.

3. Edición de las noticias, proceso que llevaban a cabo los estudiantes de manera individual.

4. Distribución del contenido a través de las redes sociales.

5. Interpretación del contenido, ya que el informativo editado se re-visionaba en clase con el fin de que los estudiantes detectasen los posibles errores y evaluasen su trabajo.

Las sesiones magistrales de la materia se sustituyeron por el visionado en común de los informativos de los respectivos grupos de prácticas, y el contenido teórico se explicaba en función de los fallos o aciertos de cada pieza. De esta forma el aprendizaje se hacía en base a la práctica que habían realizado y, con la repetición, ellos mismos aprendían a distinguir en qué fallaban y a aportar posibles soluciones.

Estas sesiones magistrales se completaron con otras impartidas en las primeras semanas, en las que se explicaron nociones teóricas, se vieron tipologías variadas de informativos y se ensayaron fórmulas de redacción, locución y edición de noticias y escaletas.

\subsection{OBJETIVOS}

El principal objetivo de la práctica fue el de conseguir las destrezas y habilidades para la producción de piezas audiovisuales de contenido informativo.

Como objetivos secundarios, se tuvieron en cuenta los siguientes:

- Adquirir destrezas comunicativas, ya que el estudiante debía aprender no solo a reconocer, sino a contar la noticia de forma eficiente. Por lo tanto, resultó fundamental la consecución de habilidades de redacción adaptada al medio, capacidad de improvisación, locución y entonación, resolución de problemas en directo, etc. 
- Desarrollar destrezas y aprender a enfrentarse a situaciones diferentes desde una experiencia cada vez mayor. Al tratarse de una práctica que se repite a lo largo del cuatrimestre los estudiantes ven mejoras en su trabajo, lo que les incentiva y motiva para conseguir resultados más favorables.

- Tomar conciencia del esfuerzo necesario para conseguir un producto de mejor calidad de cara a la exposición pública mediante las redes sociales.

- Desarrollar un espíritu crítico mediante la autoevaluación y la observación de los productos elaborados por sus compañeros.

- Aprender las rutinas de la elaboración de un informativo y aplicarlas al ritmo real de producción de una redacción de informativos audiovisuales.

- Aprender a trabajar en equipo, ya que el estudiante debía realizar colaborativamente un informativo completo que se emitía en directo.

- Controlar intelectualmente los contenidos audiovisuales que iban produciendo.

- Aprender a resolver problemas tanto técnicos como humanos en la gestión diaria de la información audiovisual.

\section{CONCLUSIONES}

Tras la experiencia de innovación docente, y a modo de conclusión, cabe decir que el aprendizaje del estudiante resultó más acelerado de lo habitual en los procesos docentes en los que las prácticas se hacen en el entorno más controlado del aula. El alumno se enfrentaba de forma repetida a su propio "miedo escénico", y la sensación de exposición al público, así como la imposición de veracidad, credibilidad y rigor en la información hacían que el estudiante tuviese la sensación de estar ante un proceso real de generación de las noticias, por lo que su propia autoexigencia se incrementó de forma notable.

A lo largo del cuatrimestre, los docentes de la materia comprobaron que los estudiantes entienden mejor los procesos cuando estos se realizan como proyectos reales, haciendo y fallando (learning by doing), que si estos se ensayan en el marco del aula, donde el ambiente 
es más conservador y todos los elementos están bajo control y son conocidos por los estudiantes.

En cuanto a los resultados académicos, cabe decir que la evaluación fue peor que en otros años académicos. No solo suspendieron más, sino que en general las notas fueron también más bajas. La autoevaluación que los alumnos hacían de sus prácticas era más dura que la de los propios profesores y consideraban muy graves sus errores en la locución y las equivocaciones en la emisión del directo, por lo que tenían tendencia a calificarse con un "aprobado" como nota, lo que bajaba su media. La evaluación de la parte teórica, en cambio, no se resintió a pesar de la ausencia de las tradicionales clases magistrales en las que se exponían estos contenidos.

El empeoramiento de los resultados académicos hizo necesario un replanteamiento de la metodología de evaluación de cara al curso siguiente. En 2018-2019 se sustituyeron los informativos en directo por la elaboración de piezas, reportajes y entrevistas realizados de manera individual, y subidos también al canal de Youtube de la asignatura. La finalidad de su elaboración era la misma, es decir, que las piezas sirviesen para su posible emisión en un programa informativo y que el estudiante expusiese su trabajo al público, pero sin la presión del directo. En cambio, como los directos sí se consideraron útiles en el proceso de aprendizaje del estudiante, ya que ayudaban a mejorar la locución y la presencia ante la cámara, continuaron realizándose como prácticas de clase no evaluadas.

Por su parte, los estudiantes consideraron en general que la práctica era interesante, que se sintieron motivados y que habían aprendido mucho con el proceso, pero también mencionaron que les resultaba estresante por la presión de la inmediatez y de la evaluación de la audiencia pública.

La exposición a través de la presentación en directo ante sus restantes compañeros de clase, así como la exhibición del informativo en las redes sociales, ayudó a los alumnos a ser conscientes de los errores cometidos y mejorar con la repetición del proceso, fase 
fundamental de los proyectos educativos basados en el aprendizaje experiencial y el learning by doing.

Asimismo, la propia realización de contenidos que forman parte del entorno social cercano al estudiante, favoreció la interrelación entre entidades, estudiantes, medios masivos y públicos (Bueno, T.; Hänninen, L., 2012). Ello hizo que los alumnos se implicasen en mayor medida en la realidad social, política y cultural de su entorno más próximo, mejorando su visión crítica del mundo y aprendiendo a interactuar con los agentes sociales del mismo, a través de la investigación sobre el tema de trabajo y la realización de entrevistas personales, necesarias para el desarrollo de la tarea periodística.

\section{REFERENCIAS}

Aguiar, B.; Velázquez, R. \& Aguiar, J. (2018) Innovación docente y empleo de las TIC en la Educación Superior. Espacios. Vol. 40 (№ 2), pp: 8-20.

Badía, A.; García, B. (2010) Incorporación de las TIC en la enseñanza y el aprendizaje basados en la elaboración colaborativa de proyectos. Revista de Universidad y Sociedad del Conocimiento, vol. 3, núm. 2, octubre, 2006, pp. 42-54.

Bueno, T.; Hänninen, L. (2012) Innovación docente con jóvenes interactivos: proyectos sociales reales y estrategias digitales para enseñar comunicación. Estudios sobre el Mensaje Periodístico. Vol. 18 Núm. especial octubre (2012), pp: 161170. DOl: https://revistas.ucm.es/index.php/ESMP/article/view/40892

Casals, M.J. (2006) La enseñanza del periodismo y las nuevas tecnologías de la información y de la comunicación. Estudios sobre el mensaje periodístico, 12, pp. 59-70.

Domingo, D., Quandt, T., Heinonen, A., Paulussen, S., Singer, J. B., \& Vujnovic, M. (2008). Participatory journalism practices in the media and beyond: An international comparative study of initiatives in online newspapers. Journalism Practice, 2(3), pp. 326-342. 
Gil-Lacruz, M.; Gracia-Pérez, M.L.; Gil-Lacruz, A.I. (2019) Learning by doing and training satisfaction: An evaluation by health care professionals. Int. J. Environ.Research Public Health, $\mathrm{n}^{0}$ 16, pp.: 1397.

Machado, E. \& Teixeira, T. (2016) Innovation as an essential part of journalism education in contemporary societies. Journal of Applied Journalism \& Media Studies, volumen 5, №. 1, 1 marzo 2016, pp. 103-116 (14).

Manfredi, J. L.; Ufarte, M. J.\& Herranz, J. M. (2019) Innovación periodística y sociedad digital: Una adaptación de los estudios de Periodismo. Revista Latina de Comunicación Social, 74, pp. 1633-1654. D0I: 10.4185/RLCS-2019-1402

Marti, J. A.; Heydrich, M.; Rojas, M.; Hernández, A. (2010) Aprendizaje basado en proyectos: una experiencia de innovación docente. Revista Universidad EAFIT, 46 (158), pp. 11-21. Recuperado de http://publicaciones.eafit.edu.co/index.php/revista-universidadeafit/article/view/743

\footnotetext{
'Aparato electrónico que refleja el texto de una noticia y que se va moviendo a la velocidad de la lectura, generalmente gracias a la ayuda de un pedal, para que el presentador pueda leerlo a la vez que da una noticia o discurso.
} 